\title{
A Case of Hydatid Cyst in the Spleen simulating an Ovarian Cyst.
}

By T. G. Wruson, M.D., Ch.M. (Syd.), F.R.C.S. (Edin.).

Assistant Gynocologist to the Adelaide Hospital, S.A.

THE recent articles, by Lieutenant-Colonel Sturmer and Dr. Taylor, in the Journal of Obstetrics and Gynacology for October, 1904, and January, 1905, on eases where enlarged wandering spleens had simulated pelvic neoplasms, have made me think it worth while to record the following case, which was sent to me last January by Dr. Evans, of Willunga, as a probable ovarian cyst.

M.C., æt. 39, the mother of 6 children, the last confinement being 11 months ago, gave the following history :- For some weeks before her last confinement (twins) she had had pain in the left lumbar region, and this pain had been present off and on ever since. In December, 1904, after doing some sweeping, she first noticed a lump in her left side, which she said came there quite suddenly. At first she had no pain, but during the last 10 days has had considerable pain round the lump, especially in the left side, and this pain was much worse after any exertion. She had weaned her baby 7 months ago, and since then the periods had been regular, and her general health good.

On examination the patient was rather anæmic, but all ber organs apparently healthy. In the abdomen was a swelling extending up to the level of the umbilicus, and mostly to the left of the mid line. The swelling was tense, gave a fluid thrill, and except low down in the left iliac region was not tender to palpation. The tumour apparently originated in the pelvis, as the fingers could be easily passed over its upper surface. No notches were to be felt.

Vaginal examination showed the uterus high up, retroverted and pushed to the right by a cystic swelling filling up the left posterior quarter of the pelvis, and which appeared to be fixed in the pelvic brim. A diagnosis of ovarian cyst was made, and the possibility of some twisting of the pedicle suggested, though in writing to Dr. Evans I remarked on the patient not having noticed such a large swelling before, and on her account of it having come on so suddenly. The abdomen was opened in the mid line, and on passing the hand round the tumour something was felt to give way on the left side, and the tumour suddenly disappeared, and a large bulging was now noticed below the left costal region. On further examination this 
was found to be a large hydatid cyst in the spleen, which had become dislocated and impacted in the pelvic brim, and the roughened area where it had been adherent on the left side could be seen at its lower pole. The spleen itself was spread on the inner and posterior aspect of the cyst, so that when fixed down in the pelvic brim the notches on the spleen looked backwards and inwards, and were therefore not palpable. The uterus was found now to have resumed its normal position, and both ovaries were normal. The first incision was closed, and a second incision made just below the left costal region, the cyst contents (over 4 pints) and the endocyst evacuated, and the cyst cavity thoroughly flushed out with saline. As much as possible of the redundant adventitious capsule was cut away, and the edges sewn up to the incision in the parietes, which was sewn up in layers, leaving an opening for a small rubber tube leading to the cyst cavity. This tube was removed at the end of 24 hours, after which the wound was dry, except for a little serous discharge, and the patient left the hospital at the end of three weeks.

In South Australia the question of hydatid cyst always intrudes itself in the differential diagnosis of abdominal tumours, but even here, hydatid cysts involving the spleen are comparatively rare, and for an hydatid cyst to develop in the spleen and cause a dislocation of that organ sufficient to simulate an ovarian cyst must be rather unusual, and I have not been able to find a record of a similar case.

The ordinary treatment of an hydatid cyst, viz., the insertion of a large drainage tube into the space left after the removal of the cyst, and subsequent washing out of this carity till it has closed up by granulation, is very frequently followed by infection of the cavity at the time of these manipulations, which very considerably lengthens the period of convalescence, and in the Out-Patient Department, one constantly sees the sinuses left after hydatid operations, which go on discharging for months. Of course, in cases where the hydatid cyst has been infected before operation, this is inevitable, but in an uncomplicated case like the above, there seems no reason why the dead space left by the removal of an hydatid cyst should not be treated in the same way as a dead space left after any other aseptic operation, and by allowing the serous discharge that collects (in the first few hours after operation) to escape, to aim at primary union of the incision. This method, though advocated by Dr. Thornton, of London, nearly 20 years ago, does not seem to have so far met with general acceptance in the treatment of these cases, though given asepsis at the operation, there seems no reason why it should not be successful. 